\title{
Vertical stacks of small InAs/GaAs self-assembled dots: resonant and non-resonant excitation
}

J. Martínez-Pastor, ${ }^{\text {a * B. Alén, }}{ }^{\text {a C. Rudamas, }}{ }^{\text {P Ph. Roussignol, }}{ }^{b}$ L. González, ${ }^{c}$ J.M. García, ${ }^{c}$.

${ }^{a}$ Instituto de Ciencia de los Materiales de la Universidad de Valencia, P. O. Box 2085, 46071 (Valencia), Spain.

'Laboratoire de Physique de la Matière Condensée de l'Ecole Normale Supérieure, 24 rue Lhomond, 75231 Paris Cedex 05, France.

' Instituto de Microelectrónica de Madrid (CNM-CSIC), Isaac Newton 8, P.T.M., 28760 Tres Cantos (Madrid), Spain.

\begin{abstract}
We have performed photoluminescence experiments in samples containing self-assembled quantum dots with different spacer layer thickness. A strong filtering effect produced by the GaAs spacer layer on the dots sizes is observed for thin spacers $(2-5 \mathrm{~nm})$. This effect produces a blue shift of the emission band from stacked dots and a simultaneous linewidth narrowing. At the same time, given the existence of a broad dot size distribution in the first layer, bigger dots evolve towards InAs cylinder-like structures, whose emission occurs at appreciably lower energies as compared to the emission band in single layer samples.
\end{abstract}

Keywords: quantum dots, photoluminescence, vertical stacks.

Corresponding author: Juan Martínez-Pastor.

Instituto de Ciencia de los Materiales de la Universidad de Valencia, P.O. Box 2085, 46071 Valencia, Spain. Fax: +34 963983633, e-mail: martinep@uv.es 
Stacking of self-assembled quantum dots QDs in multilayer structures has been proposed as an efficient mechanism to improve the performance of new devices based on them. By the one hand, stacks of QDs increase the active volume in QD lasers and hence the modal gain. Moreover, it has been demonstrated that the overall uniformity of the QD ensemble is enhanced by the stacking process, narrowing its associated emission band, and resulting in a strong benefit on the threshold current of such lasers. Early stacking experiments revealed a redshift of the QD photoluminescence (PL) and a narrowing of the emission linewidth, either by increasing the number of layers or reducing the spacer layer thickness $[1,2]$. However, recent systematic experiments in closely stacked InAs/GaAs or Ge/Si QDs show that a blueshift of the PL can be expected under certain growth conditions $[3,4,5]$. In this work we will show how both kind of features appear in QD samples with initial very broad size distributions, and how it can be explained by the self-filtering effect produccd by the spacer layer [6].

The samples studied here consist of a multilayer structure of 10 layers containing selfassembled InAs QDs (nominal thickness 2.5 ML) separated by GaAs spacer layers with different thickness $(20,10,5$ and $2 \mathrm{~nm}$ for samples $\mathrm{A}, \mathrm{B}, \mathrm{C}$ and $\mathrm{D}$, respectively), grown by MBE on GaAs [001] at a substrate temperature of $480^{\circ} \mathrm{C}$. Given the low substrate temperature used during the growth, the dots under study are particularly small, being the average height $(H)$ and width $(D)$ of 7 and $22 \mathrm{~nm}$, respectively [7]. Moreover, the QD size distribution is very broad and can be better described by a multi-modal distribution rather than mono-modal. Such a distribution follows the empirical relation $D=11.5+1.5 \mathrm{H}$, with $H$ restricted to the interval between 3 and $11 \mathrm{~nm}$, approximately. These values where taken from statistics on AFM micrographs in a typical single layer sample without GaAs capping 
layer, but grown under similar conditions [8]. PL under resonant $(840-1000 \mathrm{~nm})$ and non resonant $(514 \mathrm{~nm})$ excitation conditions is examined at low temperatures for the different samples with two different standard setups.

Figure 1 shows the PL spectra measured at low temperatures under non resonant (514 nm) and near resonant $(835 \mathrm{~nm})$ excitation. The PL of the sample with a $20 \mathrm{~nm}$ thick spacer layer is characterized by peak energies between 1.12 and $1.2 \mathrm{eV}$ under non-resonant excitation, as shown in Fig. 1(a). On the other hand, under near resonant excitation, the high energy side of the emission is enhanced, which leads to an effective blue shift of the PL peak. This effect is also observed in single layer samples, as reported in Ref. 8 and can be attributed to the enhancement of the emission of the smaller dot families from the actual size distribution. In sample $A$, the strain field cannot drive efficiently a vertical correlation for most of the dots in the buried layers [9]. Hence, the optical quality of the dot multilayer is seriously compromised (poor uniformity throughout the sample surface and broad PL bands), reproducing approximately what was observed in the single layer samples, i.e. the PL band is consistent with the multimodal distribution of dot sizes given above.

The situation is slightly different for sample B (spacer thickness $10 \mathrm{~nm}$ ), where vertical correlation is now being possible [9]. The inhomogeneity throughout the sample surface is strongly reduced, as observed in Fig. 1(b), and the PL is peaked at around $1.17 \mathrm{eV}(1.21$ eV) under non resonant (near resonant) excitation. In a previous work, we have estimated the optical transition energies corresponding to the dot size distribution given above and they will be used here for comparison [8]. Correlating the calculated energies with the PL peak positions under non resonant (near resonant) excitation for sample $\mathrm{B}$, the emission would be consistent with an average dot height around $9(7.5) \mathrm{nm}$. However, more 
important is the greater uniformity and narrower PL band $(60-70 \mathrm{meV})$ found in this case. Even for the relatively thick spacer used, where the vertical correlated dots are expected to be electronically decoupled, the strain propagation through the spacers enables a size selffiltering effect which reduces drastically the width of the size distribution of the dot ensemble along the vertical direction $[6,10]$.

Of greater importance are the changes observed in sample C (5 $\mathrm{nm}$ thick GaAs spacer layer), as shown in Fig. 1(c). In this case, two PL bands at around $1.075 \mathrm{eV}$ and $1.205 \mathrm{eV}$ dominate the emission under non-resonant excitation. The uniformity throughout the sample surface is also good, even if a change in the relative intensities of such bands is observed on different points in the sample. Under near resonant excitation conditions the PL spectrum is dominated by the high energy PL band, slightly shifted to the blue (1.23 $\mathrm{eV}$. Together with the overall enhanced optical quality, from the $20 \mathrm{~nm}$ spacer sample to the $5 \mathrm{~nm}$ one, we find two important features to emphasize: (i) the appearance of a PL band peaked at apprectably lower energies, as compared to the PL peak energies measured in the 20 and $10 \mathrm{~nm}$ spacer samples, and (ii) a blue shift of the high energy PL band in the $5 \mathrm{~nm}$ spacer sample compared once more with the PL bands in the other two samples. Both kind of features will be explained below within the framework of the broad dot size distribution present in our samples and the important role played by the GaAs spacer layer as a size filter.

As aforementioned, the average height of the uncapped dots is around $7 \mathrm{~nm}$, which means that 5 and $2 \mathrm{~nm}$ spacers are not thick enough to cover completely the dots in the buried layer. This will produce a strong coupling between vertical correlated dots in each successive layer and even a continuity of InAs, resulting in cylinder-like InAs structures [2]. 
Hence, the PL band at around $1.075 \mathrm{eV}$ observed under non resonant conditions in the $2 \mathrm{~nm}$ spacer sample, and the low energy PL band in the $5 \mathrm{~nm}$ spacer one, can be due to the neat one-dimensional confinement of carriers in such InAs cylinder-like structures. At the same time, we could say that dots giving rise to such structures should be those from the existing dot distribution in the first layer with $H$ greater than about $8 \mathrm{~nm}$ (i.e., $8-11 \mathrm{~nm}$ ). Evidently, these values can be smaller after a partial GaAs covering, because of the reorganization of the InAs material promoted by the strain fields $[2,5]$. For the $2 \mathrm{~nm}$ spacer sample, most of the dot stacks evolves towards cylinder-like structures, except a minor quantity which are observed under near resonant conditions, as revealed by the narrow PL band peaked at 1.31 $\mathrm{eV}$, as shown in Fig. 1(d).

The hypothesis depicted above for explaining the PL bands at $1.075 \mathrm{eV}$ in 2 and $5 \mathrm{~nm}$ spacer samples is also the basis for understanding the aforementioned blue shift in the PL bands associated to dot stacks (vertically aligned dots but separated by GaAs). The blueshift is clearly more important for the $2 \mathrm{~nm}$ spacer sample, where now a narrow PL line is observed at near $100 \mathrm{meV}$ above the PL band measured in the $10 \mathrm{~nm}$ spacer sample. By reducing more the spacer thickness, the vertical alignment of the dots (with more or less important electronic coupling, depending on the remaining GaAs thickness between dots) is enabled for the smaller dots of the distribution, even the smallest ones when reducing the spacer thickness up to $2 \mathrm{~nm}$. Simultaneously, due to the size self-filtering effect, we can also say that in these stacks the dot size is more uniform than in single layer samples. As aforementioned, the height of the partially covered dots can be reduced (maybe more than factor two during the self-assembling process in stacked layers $[3,4]$. So, together with the strong coupled cylinder-like structures, produced by the larger dots and emitting at lower 
energies due to the quasi-1D confinement, the remaining InAs material can contribute to small dot stacks emitting at higher energies. Both mechanisms reasonably explain the observed blueshift of the PL towards higher energies and thus, such small size stacks would be responsible of the observed PL under resonant excitation. Since the density of the smaller dots in the initial size distribution is very low as compared to those around the center of the distribution, it is reasonable to expect a PL line narrowing for the thinner GaAs spacers, as observed in Fig. 1(d) for the $2 \mathrm{~nm}$ spacer sample. In fact, when resonant excitation is performed inside the PL band we can clearly observe how the broad PL band measured in the $5 \mathrm{~nm}$ spacer sample evolves towards several narrow PL lines, as shown in Fig. 2(a), at $1.210,1.238,1.275$ and $1.314 \mathrm{eV}$. In the case of the $2 \mathrm{~nm}$ spacer sample, the PL band is formed by two clear lines at around 1.287 and $1.313 \mathrm{eV}$, independently of the excitation energy. More resonant excitation only produces a better resolution of such lines with smaller linewidth, which reaches a minimum value around $13.5 \mathrm{meV}$.

Taking as a reference the size distribution given above for single dot layer samples without GaAs covering, the PL peak energy measured for the main line in the $2 \mathrm{~nm}$ spacer sample would correspond to stacks of dots around $4.8 \mathrm{~nm}$ high and the emission at $1.287 \mathrm{eV}$ to stacks of dots around 2 monolayers higher $(5.4 \mathrm{~nm})$. In the same way, the four PL lines measured in the $5 \mathrm{~nm}$ spacer sample under resonant conditions [Fig. 2(a)] would arise also from the same kind of stacks plus other two formed by dots 6.5 and $7.3 \mathrm{~nm}$ high. That is, height fluctuations below $2 \mathrm{ML}$ within the same stack can be deduced, giving support to the hypothesis of the filtering effect of the GaAs spacer thickness on the smaller dot sizes in the stacks. 
In summary, we have investigated the effect of the GaAs spacer thickness on the emission from an ensemble of QDs whose size distribution is typically multimodal (height from 3 to $11 \mathrm{~nm})$. On the one hand, this particularity makes possible to observe emission from InAs cylinder-like structures. On the other hand, for thin GaAs spacers $(2-5 \mathrm{~nm})$, the size filtering effect makes also possible the smaller dots of the distribution to be stacked, giving rise to well resolved and narrow PL lines at higher energies if compared to that measured for InAs cylinder-like structures.

This work has been supported by the Spanish CICYT under the project no. TIC99-1035C02 and European Commission GROWTH program NANOMAT project, Contract no. G5RD-CT-2001-00545. 


\section{References:}

[1] G. S. Solomon, J. A. Trezza, A. F. Marshall, and J. S. Harris Jr., Phys. Rev. Lett. 76, 952 (1996).

[2] N. N. Ledentsov, V. A. Shchukin, M. Grundmann, N. Kirstaedter, J. Böhrer, O. Schmidt, D. Bimberg, V. M. Ustinov, A. Yu. Egorov, A. E. Zhukov, P. S. Kop'ev, S. V. Zaitsev, N. Yu. Gordeev, Zh. I. Alferov, A. I. Borovkov, A. O. Kosogov, S. S. Ruvimov, P. Werner, U. Gösele, and J. Heydenreich, Phys. Rev. B 54, 8743 (1996).

[3] H. Heidemeyer, S. Kiravittaya, C. Müller, N. Y. Jin-Phillipp, and O. G. Schmidt, Appl. Phys. Lett. 80, 1544 (2002).

[4] M. O, Lipinski, H. Schuler, O. G. Schmidt, K. Eberl and N. Y. Jin-Phillipp, Appl. Phys. Lett. 77, 1789 (2000).

[5] O. G. Schmidt and K. Eberl, Phys. Rev. B 61, 13721 (2000).

[6] J. Tersoff, C. Teichett, and M. G. Lagally, Phys. Rev. Lett. 76, 1675 (1996).

[7] L. Chu, M. Arzberger, G. Böhm, and G. Abstreiter, J. Appl. Phys. 85, 2355 (1999).

[8] C. Rudamas, J. Martínez-Pastor, A. García-Cristóbal, Ph. Roussignol, J. M. García, and L. González, Surf. Science 507-510, 624 (2002).

[9] Q. Xie, A. Madhukar, P. Chen, and N. P. Kobayashi, Phys. Rev. Lett. 75, 2542 (1995).

[10] B. Alén, J. Martínez-Pastor, J. M. García, L. González, A. Ponce, S. I. Molina, and R. García, Phys. Rev. B Rapid Comm. 65, R241301 (2002). 


\section{List of figure captions:}

Fig 1. The PL spectra under near resonant excitation $(835 \mathrm{~nm})$ are depicted by solid lines for samples with different spacer layer thickness. Dashed lines and dotted lines are PL spectra under non resonant excitation $(514 \mathrm{~nm})$ in two different points of each sample.

Fig 2. PL spectra at different resonant excitation energies for samples with $2 \mathrm{~nm}$ (a), and 5 nm (b) thick spacer layer. 




Fig 1. J. Matínez-Pastor et al. 


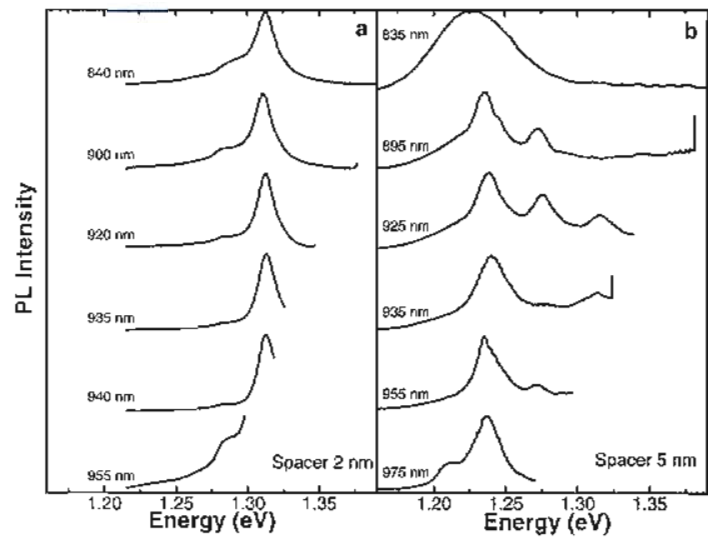

Fig 2. J. Matínez-Pastor et al. 